\title{
A Report on Midwest Regional Hospital, Surkhet
}

\author{
A team of two members from KAHS, Prof. M N Marhatta, Dean and Assistant prof. Dr Binod Aryal, \\ Gynaecologist visited the Surkhet Regional Hospital on 4th and 5th Bhadra 2074.
}

Email:marhatta@hotmail.com

\section{Objectives of visit}

- To be familiar with the Midwest Regional Hospital, Surkhet and its management

- $\quad$ To study the current status of Surkhet regional hospital in terms of Infrastructure, Human resources, clinical activities and trainings.

- To develop collaboration and cooperation between KAHS and Midwest regional hospital, Surkhet for mutual development of both the organizations.

- To upgrade clinical services, and explore the need and feasibility of various level of clinical and para-clinical educational program and researches.

\section{Method}

- $\quad$ Discussion with management

- Visit of administration / management sections and collection of data of HR and activities

- Onsite visit and observation of various wards, OPD, Emergency, pharmacy, oxygen plant, quarters etc

- Visit of New building under construction

\section{Description}

- 2074.5.4: Arrival 2.30 pm, Warm welcome and introduction

- $\quad$ Participation in Perinatal mortality morbidity review meeting being conducted

- Sort discussion about visit objectives and plan

- Letter of $\mathrm{MOH}$ on Coordination committee \& KASH letter of MBBS committee were handover by KASH dean to medical superintendent Dr. Dambar Khadka, in presence of senior Consultants, matron and officials.

- $\quad$ Short Round visit of the hospital indoor, OT, ICU, postoperative ward and emergency was made on the day.
- Evening meeting organized by medical superintendent was participated by senior consultants of the regional hospital and officials of KAHS. Healthy discussion were made on various modalities and aspects of cooperation and collaboration between KAHS and Surkhet Regional hospital.

\section{Findings}

\section{Infrastructure}

Mid-Western Regional Hospital (MWRH), is located at Kalagaun Surkhet. It is running more than 115 beds however it is approved for 50 beds by Government. Hospital is well furnished, well equipped, clean and tidy. Hospital has separate indoor, administration, OPD block and staff quarters

It has 3 operating rooms with 4 operating tables and 10 postoperative beds. ICU has 4 beds with 2 ventilators and monitors. NICU has 8 beds with radiant warmer. Paediatric ward has 8 beds.Other wards include medical, surgical, obstetric and gynecology ward. Emergency has 10 beds with central supply of oxygen and monitoring facilities. Dialysis service has just established with 4 machines.

Administration: This block has Superintendent office, Matron Office, administration, finance and record section, general store and nursing store, maintenance room and meeting hall with 60 seats.

There are nine blocks of quarters with capacity to accommodate more than 25 persons.

OPD: OPD service is in new building which is spacious having well designed examination rooms, ticket counter and patient waiting hall. Dental OPD is running by 4 Dental surgeons. Well baby clinic and physiotherapy services in OPD are impressive. 
It has own hospital pharmacy, well established lab and radio-diagnostic facilities $4 \mathrm{X}$ - rays and 4 USG and blood bank ( 2 staff) with 24 hours services.

Pharmacy: Pharmacy is run by pharmacy management committee in two places at old block and in OPD premises and is in good benefit. It is run by 6 staffs include B pharma, CMA and Diploma pharmacy.

LAB: There are separate emergency and routine laboratories. They perform most of the tests like electrolyte, LFT, RFT, CPK, Troponine, amylases, culture and all hematological tests.

Radiodiagnostic: There are 3USG machines in the hospital. 7960 patients got the service last year. Similarly there are $4 \mathrm{X}$-ray machines and 10735patients got the service in the year 2073/74.

Hospital has Oxygen plant with the facilities of central supply system and cylinders filling system. There is facility of Water guard plant for drinking water. Waste management system uses earthworm farming, biogas and pits.

Human resources: Consultants

Obstetrician and Gynecologist (rank 11) 1

Orthopedic surgeon (rank 11)

General Surgeon (rank 11)

Medical Superintendent (rank 11)

Anesthesiologist from KAHS

Orthopedic surgeon (rank 9)

Paediatrician (rank 8)

Physician MD internal Med

Radiologist (rank 8)

Gynecologist

Dental surgeon (BDS)

Medical officer

MDGP Resident

Nursing

Senior Nursing Administer

Nursing officer

Staff Nurses

ANM

AHW

\section{Supportive staff}

Laboratory is run by 8 technicians, Pharmacy is covered by 6 staffs, Blood Bank is operated by 2 staffs and there is 1 staff to run Physiotherapy.
Skilled birth attendance training (SBA) Training of 2 months period is being conducted regularly.

There is Nutrition house with 10 beds for the care of malnourished children.

\section{Activities}

Annual Data of 2073/74 (2016)

OPD Attendance -

Emergency attendance 4574

Total admission $\quad 9730$

Recovered - $\quad 9482$

Referred 72

LAMA 71

Absconded 16

Mortality - $\quad 15$

Total delivery $\quad 3466$

ND - 2590

$\mathrm{C} / \mathrm{S} \quad 653$

Vacuum delivery $\quad 175$

Breech 48

Major Surgery 5-6/day

Occupancy rate $\quad 89.03 \%$

Investigations

X-ray 10735

USG $\quad 7960$

The hospital is running various in-service training for nursing and paramedics. The hospital has been posting site of students for various graduate, postgraduate and nursing education programs.

\section{Future Plan}

- Completion of 300 bed under construction hospital within one and half year

- $\quad$ Regional lab with modern technology in full capacity is going to be operated soon.

- Various level of educational program and researches in collaboration and cooperation with KAHS.

\section{Conclusion}

The Midwest Regional Hospital Surkhet is managed very well. It is proving various basic as well as specific quality health services to the public. As a regional Centre it is running various training to the health professionals of the region. It is developing quickly and strengthening its Capacity. The center is interested to start various level of training / educational program and researches in collaboration and cooperation with Karnali Academy of Health Sciences (KAHS), Jumla. 